\title{
Quantitative Convergent Beam Electron Diffraction for Simultaneous Structure Factor and Debye Waller Factor Determination - Fitting Method Optimization
}

\author{
X. Sang, A. Kulovits, J.M.K. Wiezorek
}

Department of Mechanical Engineering and Materials Science, Swanson School of Engineering, University of Pittsburgh, 648 Benedum Hall, 3700 O Hara Street, Pittsburgh, PA 15261

Electronic structure calculations offer popular tools for the study of materials and have been applied to elucidate intrinsic properties of the isostructural tetragonal ordered phases $\gamma_{1}-\mathrm{FePd}$ and $\gamma_{2}-\mathrm{FePt}$ (Fig. 1). With Pd and Pt belonging to the same column of the periodic system of elements these phases may be useful as model systems for study of $3 d-4 d$ and $3 d-5 d$ electron interactions. Interestingly, despite their structural similarities, they exhibit differences regarding the temperature dependence of the magnetic anisotropy energy, solid solution behavior and ordering temperature. Ultimately, we intend to use quantitative convergent beam electron diffraction (CBED) in energyfiltered transmission electron microscopes (EFTEM) to provide experimental data suitable for validation of theoretical/computational findings for FePd and FePt. CBED is very attractive as it uses very small defect free specimen volumes, thereby avoiding complications associated with many other experimental methods used for intrinsic property measurements, which require use of large "real" defected samples [1]. Experimental determination of the electron density distribution by CBED requires very precise and accurate structure factor measurements [1]. This includes CBED pattern acquisition and computational refinement. Here, we used a JEOL JEM2100F with a Tridiem GIF and a probe corrected JEOL JEM 2200FS with in-column $\Omega$ energy filter for CBED experiments. We tested existing CBED structure factor refinement methods, such as the excited row and the zone axis pattern methods, since they offer somewhat complementary advantages and suffer from their different respective disadvantages [1,2].

Here we introduce a new line profile based method for pattern matching and refinements of ZAP CBED data by use of a rectangular area for refinement. We have obtained significantly improved goodness of fit, R- values, e.g. $\mathrm{R}^{\mathrm{HOLZ}}$ from 0.2 [2] to less than 0.1 (Fig. 2). Also, using special orientations with multiple excited g-vectors, e.g. Fig. 2e where we used a symmetric 4-beam orientation, allows fitting of multiple excited g-vectors. This has advantages regarding addressing the challenges associated with the need of simultaneous determination of $\mathrm{F}_{\mathrm{hkl}}$ and $\mathrm{B}$ for unknown multi-elemental phases, such as FePt and FePd. Notably, temperature factors have been reported for the elements $\mathrm{Fe}, \mathrm{Pd}$ and $\mathrm{Pt}$ in their common crystal forms, but are not known for the chemically ordered phases, $\gamma_{1}-\mathrm{FePd}$ and $\gamma_{2}-\mathrm{FePt}$ with their tetragonal structure (Fig. 1), implying need for simultaneous refinements of multiple anisotropic Debye Waller factors, $\mathrm{B}_{\mathrm{M}}$, where $\mathrm{M}=\mathrm{Fe}$ or $\mathrm{Pd}(\mathrm{Pt})$, and structure factors, $\mathrm{F}_{\mathrm{hkl}}$. . The interplay of two or three strongly excited beams and their respective $\mathrm{F}_{\mathrm{hkl}}$ and $\mathrm{B}$ can cause significant intensity changes in the pattern, which may be exploited to discriminate between possible solutions for an optimized fit. If only one g-beam is excited, frequently, intensity profiles fail to vary significantly for changes in either $\mathrm{F}_{\mathrm{hkl}}$ or $\mathrm{B}$, resulting in matches of comparable R-values for different combinations of $\mathrm{B}$ and $\mathrm{F}_{\mathrm{hkl}}$. Conversely, the conventional ZAP method requires consideration of the simultaneous interactions of more than three $\mathrm{F}_{\mathrm{hkl}}$ and $\mathrm{B}$, increasing calculation time, complicating interpretation and reducing the goodness of fit due to difficult background subtraction and distortion problems. Using Si as a test case we present comparison of the new refinement method with the two conventional methods. In the example of 
Figure 2 we fixed the structure factor(s) and optimized for the Debye Waller factor for Si. The new method improvement in R-value relative to the conventional ZAP method while retaining enough information to determine $\mathrm{B}$ confidently with a distinct minimum in the $\mathrm{R}$ vs. B curve, while the excited row method produces a shallow minimum for R-values for R vs. B.

Thus, we present a new refinement method, which retains advantages of the ZAP method, e.g. large information content per CBED pattern, and offers improved treatment of distortions and background subtraction for simultaneously fitting $\mathrm{F}_{\mathrm{hkl}}$ or $\mathrm{B}$ sensitive discs in special beam orientations using a rectangular profile. We tested this method on Si and will apply it to FePd and FePt.

We acknowledge support from the Office of Science, Office of Basic Energy Sciences, Division of Materials Sciences and Engineering, U.S. Department of Energy under contract DE-FG0208ER46545, and access to facilities of the Materials Micro-Characterization Laboratory of the Department of Mechanical Engineering and Materials Science, University of Pittsburgh and the Center of Microanalysis of Materials at the University of Illinois at Urbana Champaign.

[1] J. M. Zuo et al. Nature 401 (1999) 49-52

[2] K. Tsuda, M. Tanaka Acta Crystall. A 55 (1999) 934-954
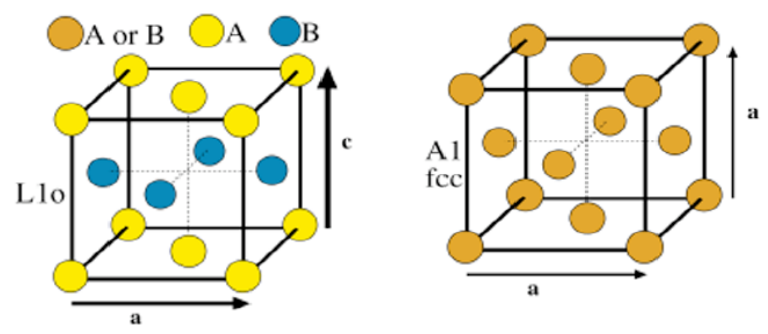

Fig. 1: Conventional unit cell representations of the chemically ordered, $\mathrm{L} 1_{0}$, and chemically disordered, A1, crystal structures of the A-B compounds FePd and FePt.
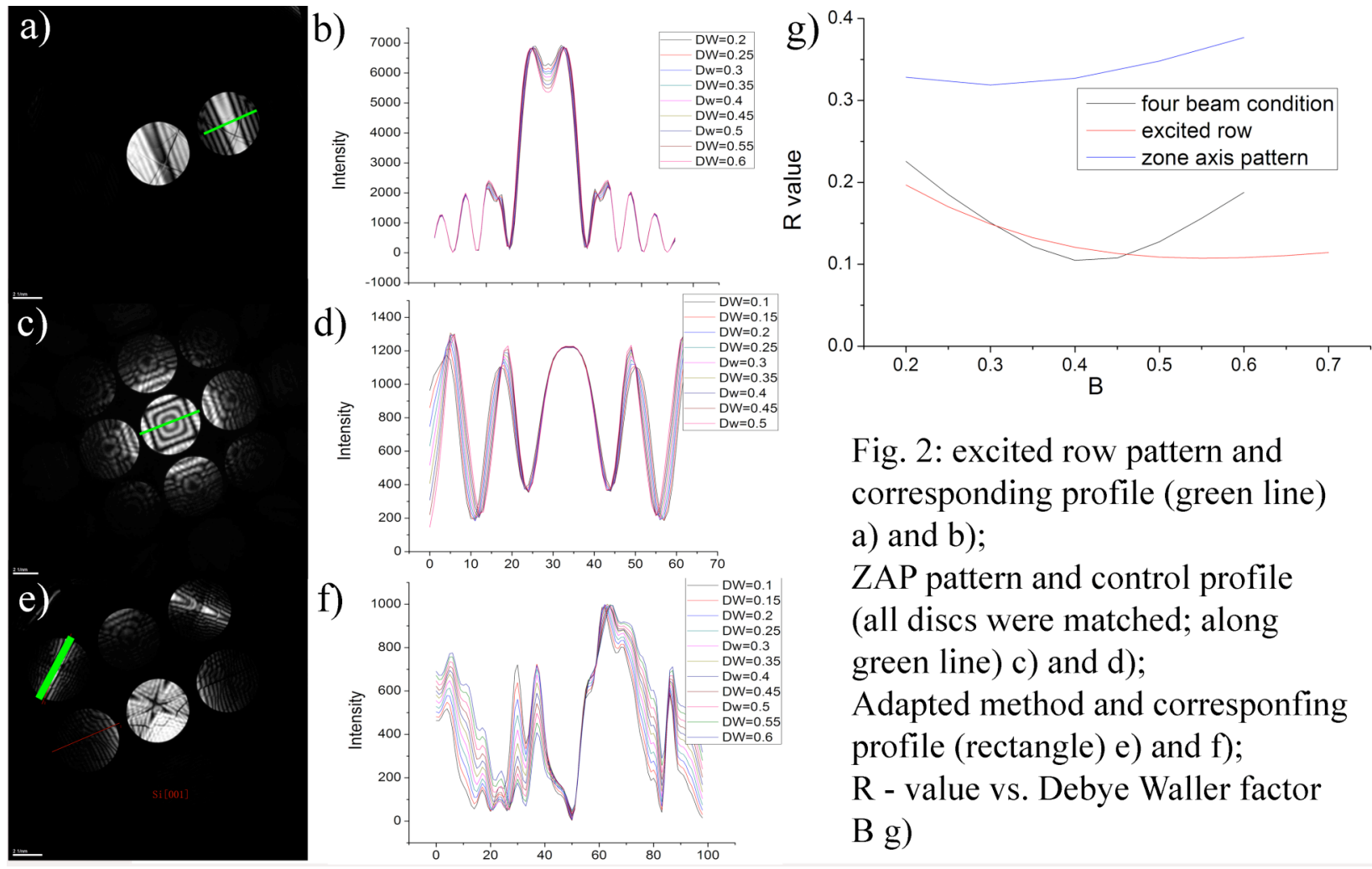

Fig. 2: excited row pattern and corresponding profile (green line) a) and b); ZAP pattern and control profile (all discs were matched; along green line) c) and d); Adapted method and corresponfing profile (rectangle) e) and f); R - value vs. Debye Waller factor $\mathrm{B}$ g) 